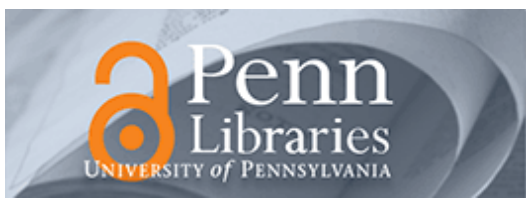

University of Pennsylvania ScholarlyCommons

Departmental Papers (Music)

Department of Music

1992

\title{
Harmonic Closure: Music Theory and Perception
}

Burton S. Rosner

Eugene Narmour

University of Pennsylvania, enarmour@sas.upenn.edu

Follow this and additional works at: http://repository.upenn.edu/music_papers

Part of the Musicology Commons, and the Music Theory Commons

\section{Recommended Citation}

Rosner, B. S., \& Narmour, E. (1992). Harmonic Closure: Music Theory and Perception. Music Perception: An Interdisciplinary Journal, 9 (4), 383-411. http://dx.doi.org/10.2307/40285561 


\title{
Harmonic Closure: Music Theory and Perception
}

\begin{abstract}
Music theorists have often disagreed about the material variables that determine the perception of harmonic closure. To investigate this controversial topic, we presented subjects with pairs of selected two-chord progressions. The subjects judged which member of each pair seemed more closed. Preferences varied across pairs of cadences and generally obeyed transitivity. Quantitative reformulation of theoretical harmonic variables permitted correlational analysis of the results. Three or four variables, including one or two that reflect learned stylistic structures, best explained our findings. Conventional harmonic factors of scale step, soprano position, and root position demonstrated surprisingly little explanatory power.
\end{abstract}

\section{Disciplines}

Music $\mid$ Musicology | Music Theory 


\title{
Harmonic Closure: Music Theory and Perception
}

\author{
BURTON S. ROSNER \\ University of Oxford \\ EUGENE NARMOUR \\ University of Pennsylvania
}

\begin{abstract}
Music theorists have often disagreed about the material variables that determine the perception of harmonic closure. To investigate this controversial topic, we presented subjects with pairs of selected two-chord progressions. The subjects judged which member of each pair seemed more closed. Preferences varied across pairs of cadences and generally obeyed transitivity. Quantitative reformulation of theoretical harmonic variables permitted correlational analysis of the results. Three or four variables, including one or two that reflect learned stylistic structures, best explained our findings. Conventional harmonic factors of scale step, soprano position, and root position demonstrated surprisingly litthe explanatory power.
\end{abstract}

$\mathrm{E}$ XPERIMENTAL psychology has the potential to clarify many basic problems in music theory (see Narmour, 1977, 1990; Rosner, 1988). One such problematic area concerns specification of the variables that determine various degrees of harmonic closure. If we knew more about the material, as opposed to the contextual, determinants underlying our perception of basic harmonic progressions, we could then compute, for example, the closure of the authentic cadence (V-I) compared with that of its plagal counterpart (IV-I). We use the term "authentic" to refer to any V-I regardless of inversion, soprano position, or scale-step motion. Most theory textbooks regard these three different variables-scale step, soprano position, and bass position - as the defining properties of authentic cadences (e.g., Christ \& DeLone, 1975, p. 211; Harder, 1977, pp. 140148; McHose, 1951, p. 145; Reynolds \& Warfield, 1985, p. 77; Sadai, 1980 , p. 135; Toutant, 1985, pp. 172-180). By "perfect authentic" we

Requests for reprints may be sent to Burton S. Rosner, Department of Psychology, Oxford University, South Parks Road, Oxford OX1 3UD, England. 
mean any V-I cadence with both chords in root position and soprano positions of either 3-8 (scale steps $\hat{7}-\hat{8}$ ) or $5-8$ (scale steps $\hat{2}-\hat{1}$ ).

Two contradictory traditions in music theory surround the problem of evaluating cadential closure. One, often thought to stem from Rameau (1726), holds that there are two dominants, one a fifth above the tonic (our dominant proper) and one a fifth below (the subdominant). To this branch of theory, such bipolarity has always suggested that V-I and IV-I cadences are more or less closurally equal. Many conventional books treat the closure of perfect authentic and plagal cadences as if they were closurally synonymous. For example, the first edition of the Harvard Dictionary of Music (Apel, 1944) presents them in this fashion. Bernstein (1937, p. 51) claims that the plagal progression is "equally as conclusive as the perfect cadence." Morris $(1946$, p. 7), in his still widely influential text in Great Britain, asserts that both V-I and IV-I are "full closes." Even today, Meyer (1989, p. 285) argues that the "authentic cadence is not intrinsically more closed than the plagal cadence."

The other tradition, promulgated by diverse theorists like Riemann, Schenker, Chailley, and Schoenberg, refuses to treat V-I and IV-I alike. This branch of theory maintains that the perfect authentic form is sui generis. Riemann $(1898 / 1977$, p. 28) says that IV-I is a much weaker progression than V-I. Chailley (1977/1986, p. 129) concurs, asserting that IV-I is a "static progression" compared with V-I. Schenker (1935/1979, 1956/1979) likewise contends that IV-I is in no way comparable to V-I since, in his natural-law view of musical syntax, the fifth degree $(V)$ in the perfect authentic cadence exemplifies an overtone progression moving back to its acoustic origin (I). Schoenberg $(1954$, p. 14) goes even further, proclaiming that "Plagal cadences ... are only a means of stylistic expression and are structurally of no importance."

Many contemporary music theorists take a more moderate stance, similar to White's $(1984$, p. 39). He says that the plagal cadence "has some of the same feeling of finality as the authentic cadence, but because the progression IV-I does not have the strong tonal implications of V-I (without its leading tone), its ability to define a tonality is considerably less." Most recent reference works (e.g., The New Harvard Dictionary of Music [Randel, 1986], The New Grove Dictionary of Music and Musicians [Sadie, 1980], and The New Oxford Companion to Music [Arnold, 1983]) adopt a similar position.

Analytical demonstrations aside, no empirical psychological evidence supports either the belief that V-I and IV-I are closurally equivalent or the belief that their qualities of finality are totally incommensurate. We will refer here to the overall closural quality of V-I, IV-I, and other types of known cadential progressions in tonal style as "harmonic schemata." 
Schenker, doubtless the most influential tonal theorist today, makes certain other claims about harmonic closure that beg for empirical substantiation. Consider his concept of a scale step, which denotes the apparently inherent scalar stability of soprano chroma within the key of a given cadence. In a manner similar to Rameau's invocation of two dominants, Schenker (1935/1979, 1956/1979, pp. 13, 14, 16), like many other German theorists, invokes two "leading tones," one involving scale steps $\hat{2}-\hat{1}$, the other, scale steps $\hat{7}-\hat{8}$. His work and that of all his many followers clearly imply that V-I with $\hat{2}-\hat{1}$ in the soprano establishes a noticeably greater harmonic closure than V-I with $\hat{7}-\hat{8}$. But as in comparing the closure of V-I with that of IV-I, no psychological evidence exists for ranking $\hat{2}-\hat{1}$ over $\hat{7}-\hat{8}$ in perfect authentic cadences.

Other general tenets of music theory concerning harmonic closure deserve empirical attention. For example, like Schenker (1906/1954, p. 217), Piston (1978, p. 186) says about the I of a V-I cadence that "Placing the third (of the chord) in the soprano usually gives less feeling of finality than having the tonic in both outside voices." He also asserts about the bass in the V-I that "The approach to the tonic by means of the first inversion of the dominant chord is generally considered a less conclusive cadential effect" (ibid.). Of course, Schenker's basic closural frameworks (the various forms of the Ursatz) give certain scale step motions a priori significance over others and preordain certain fundamental progressions over others. But do listeners apprehend these presumably intrinsic perceptual effects of soprano position and bass inversion on perceived harmonic closure? Do their perceptions of closure depend on whether the top voice of the underlying chord is 1,3 , or 5 from the root and whether the chord exists in the bass in root position, first inversion, or second inversion? (Notice that soprano position and scale step are two different ways of looking at the same melodic notes.)

In addition to harmonic schema, scale step, soprano position, and bass inversion, music theory postulates two other variables that affect the evaluation of harmonic motion. For example, theorists conventionally assert that the number of common tones between any two chords relates inversely to the strength of a given progression (see Piston, 1978, pp. 2122). Since diatonic triadic progressions whose roots lie a third apart share two common tones $(2 / 3)$, it is not unreasonable to hypothesize that VI-I and III-I are weaker and less closed than V-I and IV-I, where only one triadic tone $(1 / 3)$ is common. We designate this variable "commontoneness." Again, no psychological evidence supports these claims.

Another, less widely recognized theoretical device also might contribute to harmonic closure. It is simply the combined amount of perceived motion spanned by the two outside voices. We call this "outer-voice motion." All 
other things equal, increased amounts of outer-voice motion should strengthen perceptions of closure. Of course, Schenkerian analysis forcefully draws attention to the importance of the voice-leading framework in examining harmonic closure. In contrast, our concept of outer-voice motion merely means the combined amount of voice motion in both the bass and soprano. Although this motion relates to the concept of commontoneness, examples show that the former is not commensurate with the latter. One can find progressions having complete common-tone differentiation $(0 / 3)$ but very little outer-voice motion. And, vice versa, other progressions can have no common-tone differentiation (3/3) but can display great motion in their outside voices. Empirical evidence is lacking about the power of outer-voice motion.

One goal of this article is to address experimentally the influence of variables identified by music theory on listeners' perception of harmonic closure. Do harmonic schema, scale step, soprano position, bass inversion, common-toneness, and outer-voice motion influence judgments of harmonic closure in the ways that theory suggests they should? Since these different variables never operate in isolation, our second goal is to examine how such variables combine. For in a particular progression they may reinforce or conflict with one another. Empirically determining the effectiveness of a variable is one issue. A separate one is to discover how different harmonic factors, once proven efficacious, operate together to produce a listener's perception of closure.

To approach these questions, we presented subjects with pairs of twochord progressions. The subjects had to decide which progression sounded the more closed. Each progression in a pair was a variant on V-I, IV-I, III-I, or VI-I. We selected the progressions on the basis of their importance to harmonic theory. For reasons of simplicity and to control the parameter of melody, we avoided soprano lines that had skips and avoided progressions with any explicit dissonance, such as VII ${ }^{6}$-I.

\section{Choices of Progressions}

The five authentic cadences in Figure 1 are clearly germane to our questions. Comparisons among the progressions in Figures $1 \mathrm{a}, 1 \mathrm{~b}$, and $1 \mathrm{c}$ should indicate the importance that subjects attach to scale step. For these progressions, notice how scale step (indicated by carets) correlates with soprano position (uncareted). Comparing the cadences in Figures $1 \mathrm{~d}$ and $1 \mathrm{e}$ to the three preceding progressions and to each other should test whether listeners distinguish between inversions and root positions in the bass. These comparisons also should show whether the same soprano positions in the cadences of Figures $1 \mathrm{c}$ and $1 \mathrm{~d}$ and of Figures $1 \mathrm{~b}$ and $1 \mathrm{e}$ 


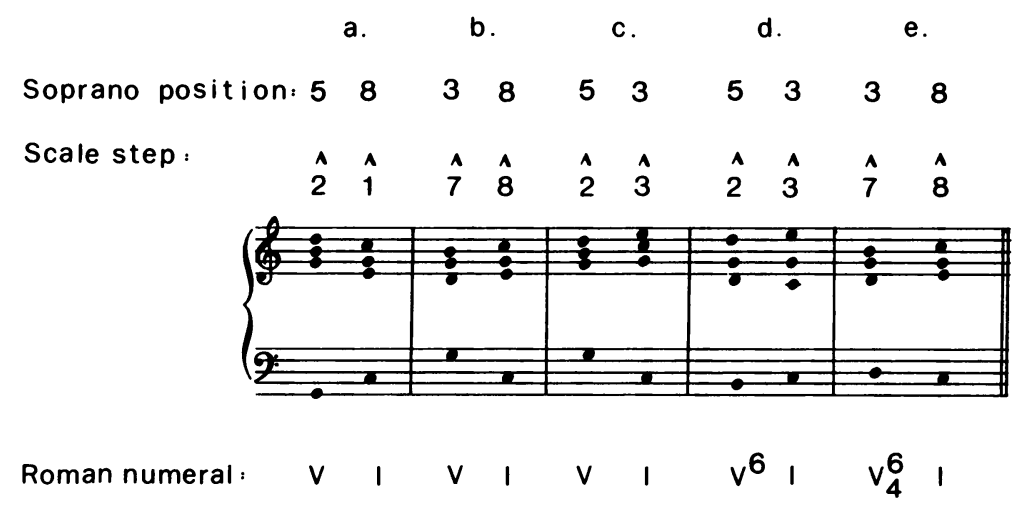

Fig. 1. Five authentic cadences.

reduce sensitivity to inversion. The progression $\mathrm{V}_{4}^{6}-\mathrm{I}$ is common within a passing-tone motion but rare as a closural cadence. Nevertheless, questions arise concerning whether listeners closurally distinguish this progression from $\mathrm{V}^{6}-\mathrm{I}$ and from the perfect authentic cadence itself (V-I).

In addition to the five dominant-tonic progressions of Figure 1, we selected seven other progressions for the experiment. They appear in Figure 2. We include the progression III-I (Figure 2a) because functional harmonic theory has frequently maintained that III can substitute for $\mathrm{V}$ (see, e.g., Riemann, 1898/1977; Tchaikovsky, 1871/1970). An obvious question therefore is whether subjects actually hear III-I as a substitute V-I cadence. Another question is whether V-I with scale step $\hat{7}-\hat{8}$ (Figure $1 \mathrm{~b}$ ) is actually perceptually stronger as a cadence than, say, $V^{6}-\mathrm{I}$ (Figure 1d). If so, will listeners then treat a III-I progression with $\hat{7}-\hat{8}$ in the soprano as closurally stronger than $\mathrm{V}^{6}$-I with the same scale step? Furthermore, will listeners distinguish the progression $\mathrm{V}_{4}^{6}$-I (Figure 1e) from III-I, whose bass and soprano motion and scale step movement closely resemble it?

Similarly, pitting the cadence in Figure $2 \mathrm{~b}$ against those in Figure $1 \mathrm{a}-\mathrm{d}$ should show whether listeners equate subdominant-tonic (plagal) motion with the closure of dominant-tonic motion. And comparisons between the cadences in Figure $2 b-d$ should demonstrate whether listeners differentiate between root position and degrees of inversion in closural instances involving the subdominant. In particular, how will listeners closurally respond to IV 4 -I in Figure 2d against IV-I or IV ${ }^{6}-I$ ? The question is interesting because both tonal and modal composers commonly employ I-IV 6 -I as a closural gesture. Of course, IV IV $_{4}^{6}$ by itself is not exactly a cadential progression but rather a double appoggiatura prolonging one chord, the tonic. We use the label "IV ${ }_{4}^{6}$-I" here only as a notational convenience, not as an adequate representation of this particular musical event itself. 
a. b. c. d.

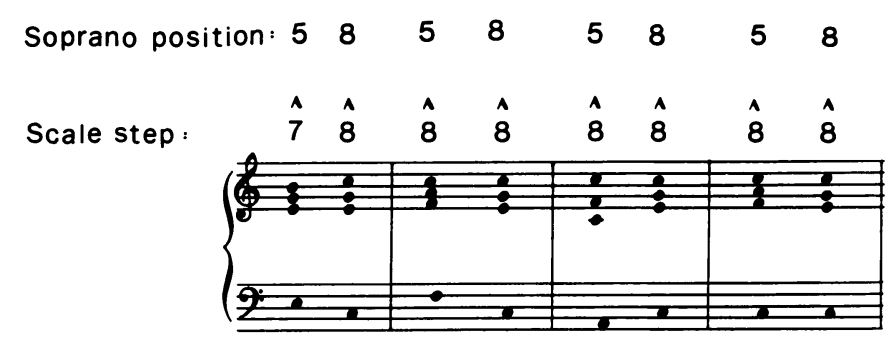

Roman numeral: III I IV I IV I $\quad I_{4}^{6}$ ।

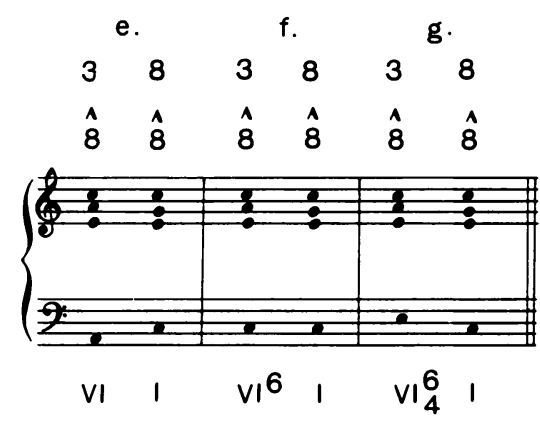

Fig. 2. Seven inauthentic progressions.

Likewise, one wants to know whether VI ${ }^{6}-\mathrm{I}$ (Figure 2f) functions as an "appoggiatural substitute" for IV 6 -I. Similarly, how will listeners evaluate $\mathrm{VI}_{4}^{6}$-I (Figure $2 \mathrm{~g}$ ) against all the other progressions involving inversions or against VI-I itself (Figure 2e)? (Again, we use figured Roman numerals only as a notational convenience.)

\section{Method}

SUBJECTS

The subject panel of the Department of Experimental Psychology at the University of Oxford provided 19 paid, volunteer listeners. They ranged in age from 20 to 40 years. None was a professional musician or had extensive musical training. Subjects were not selected for any particular interest in music or because they listened regularly to music. None had any complaints of abnormal hearing or of neurological problems.

\section{MATERIALS AND APPARATUS}

The 12 progressions in Figures 1 and 2 produce 66 different possible pairs. These 66 pairs were played by one author (EN) on a synthesizer. The order within each pair of 
progressions was arbitrarily designated as $\mathrm{AB}$. Two additional individual progressions also were produced for use as instructional examples.

All progressions were played in a rhythm of quarter note to half note at the tempo of one quarter note per second. Rhythmic cumulation ensured the durational closure of each progression. (On the psychological and analytical evidence for durational cumulation functioning closurally, see Cooper \& Meyer, 1960; Meyer, 1956, 1973; Narmour, 1977, 1990.) The progressions within a pair were played in different keys to avoid key-relation effects. The keys themselves were alternated across pairs in a mixed A/B-B/A fashion to prevent the introduction of any white-key/black-key regularity. Moreover, the first soprano tone of the second chord pair was never more than a major second's distance from the last soprano tone of the first chord pair. This avoided any effects of melodic skips between the soprano lines of the chord pairs. It also kept both chord pairs registrally close, thereby minimizing any sense that a progression on a higher pitch level was "more dynamic," achieving more closure than a progression on a lower pitch height. For the same reason, registral directions and skips in bass lines were kept as comparable as possible.

To avoid introducing unintentional biases through the addition of dynamic accents, the progressions were played on a Yamaha DX7 synthesizer using "ROM 3-B" (the "Keyboard and Plucked Group"), set on stop 16 called "Pipe Organ II." The output of the synthesizer was recorded on cassette tape.

The cassette tape was taken to the IBM UK Science Centre, Winchester, England, where the progressions were digitized at $20 \mathrm{kHz}$ after passing through a $9.7-\mathrm{kHz}$ anti-aliasing filter. Digitization produced 68 waveform files (66 pairs of progressions in $A B$ order plus the two additional instructional progressions designated as Example 1 and Example 2). We intended to use a time-domain waveform editor in order to give all quarter-note chords and half-note chords standard lengths. We quickly discovered that individual chords could not be edited to standard durations, due to properties of the Yamaha synthesizer. The waveforms of the chords changed continually and displayed mild frequency and amplitude modulation. These effects gave the output a "rich," nonmechanical sound. At the same time, they prevented any waveform editing by cutting and pasting. Attempts at such editing produced audible, unacceptable irregularities.

To insure as much durational uniformity as possible, we exploited the fact that the 66 pairs of progressions were constructed from 42 different individual progressions. The 42 unique progressions were the outcome of the key changes described above. Using the waveform editor, we produced separate waveform files for these progressions, retaining the files for instructional Examples 1 and 2. We also measured the duration of each initial chord in all 44 files. The file size gave us the duration of each progression. From these two numbers we calculated the duration of the second chord in each progression.

From the digitized waveform files, a tape-making facility produced a cassette tape that contained the desired pairs of progressions with appropriate pauses between them. The tape began with the two example progressions in certain configurations for use in conjunction with the instructions, which will be described shortly. Then came the stimuli for 132 trials. Each of the 66 pairs of progressions occurred on two different trials, once in the order $\mathrm{AB}$ and once in the order $\mathrm{BA}$. The sequence of pairs of progressions was randomized in two blocks of 66 each. Orders within pairs of progressions were randomized in a single block across all 132 trials. These two precautions were intended to eliminate any unintentional biases arising from the sequence in which the pairs of progressions were originally played.

A trial began with an announcer's speaking the trial number. Then the pair of progressions for that trial appeared twice. A silence of $2.5 \mathrm{sec}$ separated the repetition of the pair of progressions, and $1.5 \mathrm{sec}$ separated the progressions within a pair. The subject was to listen to the pair of progressions twice on each trial before responding. A silence of $7.5 \mathrm{sec}$ separated the end of the repeated pair of progressions for one trial from the beginning of the next trial. We devised this arrangement for a trial on the basis of a pilot experiment. As was stated earlier, the two progressions within a trial always appeared in different keys. The experimental tape was played to the subjects through a cassette deck, amplifier, and loudspeaker. 


\section{PROCEDURE}

Subjects were tested as a group in a 1-hr session. They first heard the instructions read aloud and followed the text on printed copies. The instructions were developed and tested in the pilot study. Subjects were told that they had to judge which member of a pair of two-chord progressions was more closed. The term "closure" was explained first by playing a highly closed progression (Example 1) and a less closed progression (Example 2). It was pointed out that the first progression is more conclusive and more satisfying than the second and would end a musical passage more clearly. The instructions went on to say that in music, closure occurs when the listener realizes that some part or all of a piece has ended. A parallel was drawn between the strength of signs of closure and punctuation in writing: "The most strongly closed progressions say that a piece has finished. This is like the words, 'THE END,' at the conclusion of a story. Less closed chord progressions in music act like a full stop (a period) at the end of a sentence, signalling that one thought is complete and a new one will follow. Still less closed progressions behave like semicolons and tell you that one complete thought will be followed by a closely related one. Just as a writer must use punctuation marks correctly, a composer must get his or her signs of closure right."

The subjects were next told about the structure of each trial. They were instructed to respond on their answer sheets only after the second presentation of the pair of progressions for a trial. If the first progression in a pair seemed more closed, they were to mark the first of two letters in the row numbered for that trial on the answer sheet. If the second progression seemed more closed, they were to mark the second of the two letters. The instructions stressed that some judgments might be difficult but that an answer should be given on each trial, even when it just seemed a guess.

The experiment was then run with a break of a few minutes after the sixty-sixth trial. At the end of the experiment, subjects were debriefed. They reported no particular difficulties in making the judgments. Results were scored by means of an interactive Pascal program.

\section{Results}

\section{CHORD DURATIONS}

Measurements showed that the first chords in the 44 different progressions (including the two examples) lasted a mean of $1.05 \mathrm{sec}$ with a standard deviation of $0.05 \mathrm{sec}$. The second chords lasted $2.45 \pm 0.07 \mathrm{sec}$. The quarter-note initial chords were therefore played as intended. The half-note concluding chords, however, exceeded their intended duration on average by about $20-25 \%$. The product-moment correlation between first and second chord durations was -0.59 , differing significantly from zero $[t(42)=2.49, p<0.02]$.

These results show that the two chords in the progressions were played in a 1:2.5 durational ratio rather than in the intended $1: 2$ ratio. The actual durational ratio and the correlation between durations with a progression may have resulted from playing each progression in isolation. The correlation indicates that shorter than average durations for first chords were followed by second chords with longer than average durations. This could have heightened the effect of durational cumulation. The standard de- 
viations for the individual chord durations, however, were relatively small, less than $5 \%$ of the average durations. Any effect due to the negative correlation between first and second chords would therefore have been minimal.

\section{PREFERENCE RESPONSES}

In each of the original 66 progressions, the first member had previously been arbitrarily designated as A and the second as B. We cumulated across the 19 subjects the number of preferences for $A$ within each of the two orders $\mathrm{AB}$ and $\mathrm{BA}$. Using a matched-sample $t$-test, we compared the number of such responses for first as against second appearances of a pair, without regard to order within a pair. The mean difference across times of appearances was 0.17 . This is not significant $[t(65)=0.37, p<0.72]$. We next compared the number of preferences for progression $A$ in the $A B$ order against the number for that progression in the BA order, without regard to time of appearance. The difference between responses to the two orders was significant $[t(65)=-3.09, p<0.003]$. The sign here indicates that subjects were somewhat biased toward choosing the second of a pair of progressions as more closed than the first.

This effect is readily explained. Silence itself is a closural marker in music, and the silence that followed the second cadence in a pair of progressions was always longer than that at the end of the first cadence. The bias, however, was not very large. The mean difference between orders was -1.3 , compared to the worst possible outcome of -19.0 . We therefore combined the preference numbers for progression $\mathrm{A}$ in each pair across the two orders, obtaining an overall preference score.

The maximum possible preference score was therefore 38 . If the obtained score for a pair of progressions was 19 or greater, progression A was taken as preferred and was renamed as progression $X$, while progression $\mathrm{B}$ was renamed as $\mathrm{Y}$. The obtained score became the preference score, $N(X p r e f Y)$, for each such pair. If the obtained score was 18 or less, progression $\mathrm{B}$ was taken as preferred and was renamed as progression $\mathrm{X}$, while progression $\mathrm{A}$ was renamed as $\mathrm{Y}$. In this case, the dependent variable $N(X p r e f Y)$ was derived by subtracting the originally obtained score from 38.

Dividing each preference score by 38 transformed it into a preference probability ranging between 0.50 and 1.00 . A probability of 1.00 means a perfect preference for the $\mathrm{X}$ progression of a pair over the $\mathrm{Y}$ progression; a probability of 0.50 indicates no preference. Table 1 shows for each pair of progressions the probability with which progression $\mathrm{X}$ was preferred as more closed to progression $\mathrm{Y}$. The progressions are indicated by the usual Roman numerals, along with scale steps for the first three $\mathrm{X}$ progressions and for all $\mathrm{Y}$ progressions. 
TABLE 1

Probability of Judging Progression X More Closed Than Progression Y

\begin{tabular}{|c|c|c|c|c|c|c|c|c|c|c|c|c|}
\hline \multirow[b]{2}{*}{ Progression $\mathrm{X}$} & \multicolumn{12}{|c|}{ Progression $Y$} \\
\hline & $\begin{array}{l}\hat{2}-\hat{1} \\
\text { V-I }\end{array}$ & $\begin{array}{l}\hat{7}-\hat{8} \\
\text { V-I }\end{array}$ & $\begin{array}{l}\hat{2}-\hat{3} \\
\text { V-I }\end{array}$ & $\begin{array}{c}\hat{2}-\hat{3} \\
V^{6}-I\end{array}$ & $\begin{array}{c}\hat{7}-\hat{8} \\
V_{4}^{6}-I\end{array}$ & $\begin{array}{l}\hat{7}-\hat{8} \\
\text { III-I }\end{array}$ & $\begin{array}{c}\hat{8}-\hat{8} \\
\text { IV-I }\end{array}$ & $\begin{array}{c}\hat{8}-\hat{8} \\
I^{6}-I\end{array}$ & $\begin{array}{c}\hat{8}-\hat{8} \\
\text { IV }_{4}^{6}-\mathrm{I}\end{array}$ & $\begin{array}{c}\hat{8}-\hat{8} \\
\text { VI-I }\end{array}$ & $\begin{array}{c}\hat{8}-\hat{8} \\
\text { VI }^{6}-\mathrm{I}\end{array}$ & $\begin{array}{c}\hat{8}-\hat{8} \\
\mathrm{VI}_{4}^{6}-\mathrm{I}\end{array}$ \\
\hline$\hat{2}-\hat{1} \quad$ V-I & - & 0.50 & 0.60 & 0.95 & 0.58 & 0.89 & 0.92 & 0.95 & 0.87 & 0.87 & 0.82 & 0.84 \\
\hline$\hat{7}-\hat{8} \quad$ V-I & & - & 0.50 & 0.63 & & 0.79 & 0.92 & 0.92 & 0.95 & 0.82 & 0.89 & 0.76 \\
\hline$\hat{2}-\hat{3} \quad$ V-I & & & - & 0.87 & 0.68 & 0.84 & 0.92 & 1.00 & 0.82 & 0.82 & 0.87 & 0.95 \\
\hline$V^{6}-I$ & & & & - & & 0.76 & 0.82 & 0.89 & 0.92 & 0.87 & 0.76 & 0.76 \\
\hline$V_{4}^{6}-I$ & & 0.53 & & 0.53 & - & 0.87 & 0.79 & 0.97 & 0.87 & 0.95 & 0.92 & 0.89 \\
\hline III-I & & & & & & - & 0.60 & 0.71 & 0.58 & 0.55 & 0.68 & 0.53 \\
\hline IV-I & & & & & & & - & 0.58 & 0.50 & & 0.68 & 0.50 \\
\hline IV6-I & & & & & & & & - & & & 0.71 & 0.74 \\
\hline$I^{6} V^{6}-\mathrm{I}$ & & & & & & & & 0.53 & - & 0.63 & 0.60 & \\
\hline VI-I & & & & & & & 0.53 & 0.71 & & - & 0.55 & \\
\hline$V^{6}{ }^{6}-I$ & & & & & & & & & & & - & \\
\hline $\mathrm{VI}_{4}^{6}-\mathrm{I}$ & & & & & & & & & 0.60 & 0.58 & 0.60 & - \\
\hline
\end{tabular}

NOTE. Italicized values significant at the 0.02 level.

Most of the preference scores appear above the major diagonal of Table 1. Only eight appear below the major diagonal, suggesting that preferences generally obeyed transitivity. More careful investigation of transitivity, however, requires assessment of the statistical significance of the preference probabilities in Table 1 .

On a null hypothesis of no preference within a given pair of progressions, the true probability of a preference is 0.50 . The standard error of an observed preference therefore is $.5 / \sqrt{38}$ or .08 . Using the normal distribution and setting $\alpha$ at 0.02 for a one-tailed test, a preference probability must equal at least 0.67 in order to be significant. An observed value below 0.67 indicates no preference. Since numerous tests were necessary, we made $\alpha$ conservative. The significant preferences in Table 1 are italicized. Such preferences occurred for 44 of the 66 pairs of progressions. Preferences for the remaining 22 pairs were not significant.

The most glaring failure of transitivity in Table 1 concerns the VI-I and IV ${ }^{6}$-I progressions. The preference score of 0.71 shows that the former was judged significantly more closed than the latter. But the IV6-I progression was preferred for closure over both inversions of VI-I, while VI-I itself was not judged more closed than its two inversions. Six additional intransitivities exist within the following triplets of progressions: $\{\mathrm{V}-\mathrm{I}$ with $\hat{2}-\hat{1}$ in the soprano, $\left.\mathrm{V}^{6}-\mathrm{I}, \mathrm{V}_{4}^{6}-\mathrm{I}\right\},\left\{\mathrm{III}-\mathrm{I}, \mathrm{IV}-\mathrm{I}, \mathrm{IV}^{6}-\mathrm{I}\right\},\left\{\mathrm{III}-\mathrm{I}, \mathrm{IV}^{6}-\mathrm{I}, \mathrm{IV}_{4}^{6}-\mathrm{I}\right\},\{\mathrm{III}-\mathrm{I}$, VI-I, VI $\left.{ }^{6}-\mathrm{I}\right\},\left\{\mathrm{III}-\mathrm{I}, \mathrm{VI}^{6}-\mathrm{I}, \mathrm{VI}_{4}^{6}-\mathrm{I}\right\}$, and $\left\{\mathrm{IV}-\mathrm{I}, \mathrm{VI}^{6}-\mathrm{I}, \mathrm{VI}_{4}^{6}-\mathrm{I}\right\}$. It is striking that every intransitivity involves one or two progressions with inversions, while only one intransitivity involves V-I cadences. 
Of the 66 pairs of progressions, 50 used different root progressions for the two chords of each pair. Table 2 shows that root effects explain most of the preferences. A V-I progression was always significantly preferred to any alternative, III-I, IV-I, or VI-I. Among the latter three types of progressions, no equally strong pattern of preferences emerged. Weaker patterns, however, do appear. Plagal cadences and VI-I progressions were never preferred to III-I chord pairs. The number of pairs that included the III-I progression, however, was small. With one exception (VI-I preferred to IV6-I), VI-I progressions were never preferred to plagal cadences. (For VI-I > IV-I, the nonsignificant preferences appear on the preceding line of Table 2 for IV-I > VI-I.)

Sixteen pairs of progressions had the same root relation for each pair of chords. Both progressions were V-I $(n=10)$, VI-I $(n=3)$, or IV-I $(n=3)$. In 10 cases, root position was compared with first or second inversion. Table 2 shows that an inversion was never preferred over root position within the same class of progressions, but the preference for root position was not very strong. In three other pairs, no significant effect of first over second inversion appeared. In the three remaining pairs of progressions, which were all V-I, differences in scale step (and soprano position) generated nonsignificant preferences.

\section{Discussion}

Two-thirds of the pairs of progressions generated significant preferences. For the most part, the pattern of preferences over all 66 pairs obeyed transitivity, although one particularly noticeable failure occurred among

TABLE 2

Preferences for Progressions

\begin{tabular}{lcc}
\hline & \multicolumn{2}{c}{ Number of Preferences } \\
\cline { 2 - 3 } Direction of Preference & Significant & Nonsignificant \\
\hline Root effects & & \\
V-I > III-I & 5 & 0 \\
V-I > IV-I & 15 & 0 \\
V-I > VI-I & 15 & 0 \\
III-I > IV-I & 1 & 2 \\
III-I > VI-I & 1 & 5 \\
IV-I > VI-I & 3 & \\
VI-I > IV-I & 1 & 7 \\
Inversion effects & & 3 \\
Root > inversion & 3 & \\
First > second & 0 & \\
\hline
\end{tabular}


seven cases of intransitivity. Root progressions apparently explain the bulk of the preferences. A striking outcome of this experiment is the preference for V-I progressions over all other root sequences. This result supports Schenker's views on the status of the perfect authentic cadence. Rootposition effects also occurred. Inversions were never preferred to root position within a class of cadences, and second inversions were never preferred to first inversions. These inversion effects, however, are not very strong. Inversion does not seem to be a major factor in determining closure, compared to harmonic schema (known cadential progressions). Scale step and its correlate, soprano position, had no clear influence on the perception of closure. Nor could we detect any effect of common-toneness. In view of the assertions of conventional music theory, the results on inversion, soprano position, and common-toneness are surprising.

The overwhelming effect of learned cadential patterning (harmonic schemata) on the preference judgments, however, may obscure any influences of other harmonic variables on the results in Table 1 . In order to explore this possibility, we reformulated all variables in quantitative terms. This permitted use of correlational methods of analysis, which are sensitive to the quantitative spread of preferences and to quantitative influences of predictor variables.

\section{QUANTIFICATION OF PARAMETRIC HARMONIC VARIABLES}

We previously described six different harmonic variables that could influence judgments of closure. Two of them represent the effect of tonal style structures. They are harmonic schema (conformance to a known cadential progression in tonal music) and scale step (inherent tonal stability of various chroma). Another stylistic variable, contextual dissonance, will also be characterized. The other four previously described variables are not necessarily dependent on a knowledge of tonal style. They are soprano position, root position, common-toneness, and outer-voice motion. Two other variables similar to them will be introduced, namely, melodic motion and modal differentiation between chords. We will show how to represent these nine variables on ordinal scales.

Music theory holds that authentic and plagal cadences frequently function as closural signs. As we have seen from Piston and Schenker, theory conceives harmonic closure partly in terms of soprano position and bass position. Thus, the closural strength of a tonic following a dominant (V-I) or a subdominant (IV-I) is supposedly greatest when either the soprano, the bass, or both constitute the root of the chord. Hence, chords in the soprano position of the third or fifth are more open than chords in the position of the root. Music theory also considers first-inversion chords ("bass position of the third") and second-inversion chords ("bass position 
of the fifth") less stable than root-position chords ("bass position of the tonic").

\section{Soprano Position}

From these suppositions, it is easy to construct ordinal measures of harmonic motion. If the soprano in the position of the root $(8)$ is more stable than that of the fifth (5), and the position of the fifth more stable than that of the third (3), then the least closed soprano motion in a progression is $5-3$, with the next most closed being 5-8, and the most closed, 3-8. These positions are assigned values of 1,2 , and 3 , respectively, while a soprano position of 8-8 constitutes nonmotion and receives a value of 0 . These values are parallel to current notions of harmonic closure. A soprano motion of 5-3 typically appears over the authentic cadence (V-I with scale-steps $\hat{2}-\hat{3}$ in the soprano); $5-8$ occurs over V-I with $\hat{2}-\hat{1} ; 3-8$ appears over V-I with $\hat{7}-\hat{8}$; and the most common plagal cadence (IV-I) has 5-8 with $\hat{8}-\hat{8}$. Figures 1 and 2 provide notated illustrations of soprano position, symbolized henceforward as SPOS.

\section{Bass Position}

In the bass, the most stable chord consists of one in root position (five-three). The first-inversion chord (six-three) is less stable than this but is presumably somewhat more stable than a second-inversion chord (sixfour). Therefore, a five-three to five-three progression will be the least differentiated and the least convincing as a sign of closure, followed by six-three to five-three and six-four to five-three. We assign to these bassposition configurations values of 1,2 , and 3 , respectively. This variable is symbolized as BPOS. Figures 1 and 2 provide examples of different bass positions.

\section{Common-Toneness}

The number of common tones between any two chords is said to relate inversely to the strength of a given progression. All other things being equal, the strongest diatonic harmonic motions should be progressions whose roots lie a second apart, since the chords involved share no common tones $(0 / 3$, e.g., the half cadence of IV-V or the deceptive cadence of V-VI). On the opposite side of the spectrum, the weakest possible progressions should be those where all tones are shared $\left(3 / 3 ;\right.$ e.g., I-I $\left.{ }^{6}\right)$. The progressions VI-I and III-I are 2/3, while V-I and IV-I are $1 / 3$. The degree of commontoneness (symbolized $C T$ ) therefore ranges from least differentiated to most differentiated: $3 / 3,2 / 3,1 / 3,0 / 3$. These $C T$ configurations receive values of $0,1,2$, and 3 , respectively. The progressions used in this experiment had $C T$ values of 1 or 2 only. 


\section{Outer-Voice Motion}

Another, less commonly recognized criterion for evaluating harmonic closure is outer-voice motion (symbolized OVM). This is simply the overall voice motion spanned in the bass and soprano. As stated earlier, it is different from common-toneness. We measure OVM in progressions by adding up the intervallic distance travelled by both outside voices. In so doing, we recognize the tritone as the maximum intervallic width, since, according to conventional harmonic theory and psychological evidence (Balzano \& Liesch, 1982), listeners harmonically (but not melodically) hear all larger intervals (fifths, sixths, and sevenths) as inversions of smaller ones at the octave (fourths, thirds, and seconds, respectively). These inversions are made as necessary in each outer voice before the results are summed.

In the V-I progression in Figure 1a, therefore, the summed, outer-voice motion measures a perfect fifth (P5), not a major sixth (M6), despite the skip of the fifth in the bass: P5 (inverted) + M2 (the major second in the soprano) $=\mathrm{P} 4+\mathrm{M} 2=\mathrm{P} 5$. The larger the summed interval representing outer voice activity, the more closed the progression. Accordingly, summed intervals of unison, minor second, major second, minor third, major third, perfect fourth, and tritone receive values of 0 through 6 , respectively.

The operation of addition here is admittedly incomplete since it ignores motion in the inside voices. Nevertheless, we assume that the outer framework between soprano and bass is considerably more salient to the ear than the melodic span of the inside voices. This assumption is consonant with work on auditory streaming (Bregman \& Campbell, 1971; Bregman \& Dannenbring, 1973; McAdams \& Bregman, 1979). Presumably, outside voices stream more easily than inside ones. Moreover, outer-voice differentiation is quite convincing in theoretical comparisons of closural progressions. The variable OVM suggests, for instance, why one could argue that the perfect authentic cadence (V-I, with a descending M2 in the soprano) is more closed than the plagal cadence (IV-I, with a unison in the soprano). The outer-voice motion (OVM) in the former is slightly greater than that of the latter (P5 vs. P4).

We turn next to two possible criteria of harmonic closure that we have not previously discussed. They are modal differentiation between chords, and melodic motion.

\section{Modal Differentiation between Chords}

Modal differentiation between chords indicates whether a diatonic progression in triadic harmony entails either two major chords, two minor chords, or a mixture of the two types. We argue here that major chords 
are more internally stable than minor chords, all other things being equal. Then, given the same closural motion of position in the soprano, a progression involving a minor/major chord configuration would seem to create more closure than a progression displaying the nondifferentiation of major/major or minor/minor. In this supposition, however, we depart from conventional harmonic theory. Although categorically recognizing the importance of major and minor chords, no textbooks known to us invoke modal differentiation between chords, symbolized as $M D C$, as a possible criterion for evaluating harmonic closure. Nevertheless, we rank nondifferentiation (major/major or minor/minor) as 0 and differentiation (major/minor or minor/major) as 1 . Therefore, $M D C$ has just two steps.

\section{Melodic Motion}

Another possible factor controlling the perception of harmonic closure is melodic motion (symbolized MM). By melodic motion we mean the actual interval in the soprano, in addition to its summed contribution to outer-voice motion (OVM). The variable of melodic motion $(M M)$ is separate from and not to be confused with that of outer-voice motion, where any interval larger than a tritone in either the bass or the soprano as an octave inversion is summed into one overall representation of differentiation. In melodic motion ( $M M)$ no limitation exists on the size of the interval hypothesized to affect the strength of harmonic closure. A major sixth is a major sixth and never a minor third.

Obviously, MM and OVM must correlate to some degree. A comparison of authentic and plagal cadences, however, shows why invoking the separate criterion of melodic motion $(M M)$ may be necessary. The variable of outer-voice motion $(O V M)$ says that a V-I progression with a major second in the soprano and with scale step $\hat{2}-\hat{1}$ is not very different from a IV-I progression with a unison and with scale step $\hat{8}-\hat{8}$ (only a M2: P5-P4). Yet it seems clear that the melodic motion (MM) of the major second in the soprano in the V-I cadence is considerably more "dynamic" than the "static" melodic motion of the unison in the IV-I cadence. The actual melodic interval in the topmost voice imparts a "dynamic quality" to harmonic progressions quite apart from the salient overall framework. Since the progressions in our experiment did not involve skips, they permit only three levels of melodic motion. These are unison, minor second, and major second, which receive values of 0,1 , and 2 , respectively.

\section{QUANTIFICATION OF STYLISTIC HARMONIC VARIABLES}

The variables identified so far lack any acknowledgment of the effect of style structures (Narmour, 1977, 1990), which play such an important role in music theory. When evaluating closure, listeners presumably invoke 
learned harmonic structures as stylistic schemata. Such schemata come into play when the stimulus displays a sufficient number of featured properties to activate them. This process relies on previously learned stylistic patterns and should be central to closural evaluation. It also should control expectation to some extent. Since sensitivity to schematic features is a matter of experience, schemata are largely culturally determined phenomena.

What are the stylistic schemata of harmonic closure? Three come to mind: root progressions of known cadences in tonal music, scale step, and contextual dissonance. Table 1 seems dominated by the power of the first of these three factors.

\section{Root-Progression Schema}

The typical authentic and plagal cadences found in tonal music and codified by music theorists for generations obviously constitute empirical style structures. But many progressions mimicking such common cadences possess enough features to function as closural signs. Consider, for example, the two progressions in Figure 3. The first is a perfect authentic cadence. Although strictly speaking in terms of Roman numerals the second progression is III $^{6}-\mathrm{I}$, its satisfactory use as a cadential substitute for V-I throughout works from Mozart to Chopin clearly attests to the perceptual power of schemata (see, e.g., the end of Chopin's Etude, op. 25, no. 1). With the exception of the "escape-tone" soprano line, III'-I resembles V-I so closely that its combined cadential features suppress the quality of the mediant chord and the property of inversion.

Experience provides listeners with harmonic schemata such as authentic or plagal cadences. Listeners will tend to hear V-I compared with, say, III $^{6}$-I as a more closed progression simply because V-I completely conforms to previously experienced instantiations of cadential closure. Put another way, perfect-authentic root progressions of V-I constitute stronger closural schemata than progressions such as III $^{6}$-I because the former are

a.

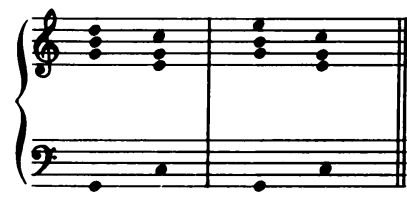

$$
\text { V } \quad 1 \quad 111^{6} \quad \text { । }
$$

( $\quad$ I $)$

Fig. 3. A schematic resemblance between two progressions. 
used far more commonly than the latter as cadential progressions in Western tonal style. Given a III ${ }^{6}$-I progression that in some ways schematically mimics V-I, the listener will perceive considerably more harmonic closure than would be expected from the variables of soprano position, bass inversion, common-toneness, modal differentiation, and melodic motion. Some additional perception of harmonic closure occurs via structural affiliation, as the example of III $^{6}-\mathrm{I}$ shows.

We will symbolize the schematic quality of known cadential root progressions as $\mathrm{SCH}$. The results in Table 1 suggest that IV-I, III-I, and V-I root sequences receive ordinal values of 1,2 , and 3, respectively. A VI-I progression is not a known cadential schema, so it receives a value of 0 . Nonzero values for authentic and plagal cadences, with the former rated more highly, reflect the roles of these cadences in much Western music.

\section{Scale Step}

Invocation of style need not be structure-specific, in the sense of calling up overt cadential representations. Certain other highly general empirical influences may affect harmonic closure. Chief among these is what is known in tonal music theory as scale step (symbolized SS). Scale step refers to the hierarchical qualities of stability and instability that inhere in the individual pitches in tonal style. Scale step is a structural phenomenon, since in certain contexts diatonic tones appear to occupy fixed positions in a spatial representation (Krumhansl \& Shepard, 1979). Listeners who know tonal style in general seem to hear the goal notes of the tonic, mediant, and dominant as more stable than the nongoal notes of the supertonic, submediant, and leading tone. Much recent work attests to the cognitive reality of this phenomenon (Castellano, Bharucha, \& Krumhansl, 1984; Krumhansl, 1979, 1983), although disputes continue over how scale-step recognition is established and the extent to which such recognition pervades the perception of a given musical composition (Butler, 1989, 1990; Cuddy \& Badertscher, 1987; Krumhansl, 1990). Howsoever these disagreements are eventually resolved, the listener's sense of scale step is purely learned. Neonates do not innately recognize this aspect of tonal style.

The hierarchical nature of scale step enables us to rank various closural motions. Given the linear or lateral voice-leading constraints that limit our progressions to registral ascent, descent, or pitch repetition, any motion in the soprano to the goal notes of $\hat{1}$ or $\hat{8}$ will create the strongest closure (e.g., $\hat{2}-\hat{1}, \hat{7}-\hat{8})$, and any motion to $\hat{3}$ the next strongest (e.g., $\hat{2}-\hat{3})$. A lack of motion $(\hat{8}-\hat{8}$ or $\hat{1}-\hat{1})$ does not contribute to closure. In the soprano, we therefore rank $\hat{8}-\hat{8}$ and $\hat{1}-\hat{1}$ as $0, \hat{2}-\hat{3}$ as 1 , and $\hat{2}-\hat{1}$ and $\hat{7}-\hat{8}$ as 2 . The progressions chosen for this experiment do not allow $\hat{4}-\hat{5}$ or $\hat{6}-\hat{5}$ motions. 
One must not confuse the empirical property of scale step (symbolized $S S$ ) with the theoretical and thus somewhat rationalistic concept of soprano position (SPOS). Both perfect authentic and perfect plagal cadences display the soprano position (SPOS) of 5-8, but the V-I progression has a scale-step motion $(S S)$ of $\hat{2}-\hat{1}$ or $\hat{7}-\hat{8}$ in contrast to the completely static $\hat{8}-\hat{8}$ or $\hat{1}-\hat{1}$ of the IV-I progression. The conventional notation of carets over the scale steps keeps SS separate from SPOS. Obviously, SS must correlate with $O V M$ and $M M$ to some degree.

\section{Contextual Dissonance}

The last construct to concern us is contextual dissonance (symbolized $C D S$ ). Contextual dissonance is a style structure in that certain progressions appear in retrospect to mimic the resolution of dissonance, thus affecting closure. Such a stylistic sense of dissonance is learned, since nothing in the chord is actually acoustically dissonant (i.e., no seconds, sevenths, or tritones are present). Context in this sense is stylistically schematic in that listeners presumably recognize retrospectively that certain inversions in particular progressions are more unstable than the prospective variable of bass position (BPOS) alone would suggest.

Among the various chord pairs, perhaps the clearest case of contextual dissonance $(C D S)$ is the progression labelled as $\mathrm{VI}^{6}-\mathrm{I}$ in Figure $2 \mathrm{f}$. There is nothing dissonant about the vertical intervals in a first-inversion chord. Yet many would hold that the harmonic, vertical sixth in this progression sounds in retrospect like an appoggiatura. The same property seems retrospectively to inhere in the second-inversion chords found in the progressions $\mathrm{V}_{4}^{6}-\mathrm{I}, \mathrm{VI}_{4}^{6}-\mathrm{I}$, and $\mathrm{IV}_{4}^{6}-\mathrm{I}$. Music theory recognizes no retrospective contextual dissonance, however, in the first-inversion chords in the progressions of $\mathrm{IV}^{6}-\mathrm{I}$ and $\mathrm{V}^{6}-\mathrm{I}$. For these, the prospective variable BPOS adequately captures the effect of the inherent instability of the inverted chords on harmonic closure. The variable of $C D S$ obviously is binary. Presence of contextual dissonance therefore receives a rating of 1 , and its absence is rated 0 .

\section{CORRELATIONAL ANALYSIS}

We assigned values for the nine harmonic variables to the individual cadences in each pair of progressions. For each variable, we next subtracted its value for the less preferred progression from the value for the more preferred one. (Where no preference whatever had been shown for a given pair, we had arbitrarily designated one member as preferred.) This yielded nine sets of differences, designated SPOSD, BPOSD, CTD, 
OVMD, MDCD, MMD, SCHD, SSD, and CDSD to compare against the dependent variable $N(X p r e f Y)$, the number of times progression $\mathrm{X}$ was judged more closed than progression $\mathrm{Y}$.

Multiple regression analysis would seem appropriate for examining the contributions to the preference judgments from the nine harmonic criteria for closure. This method, however, assumes homoscedasticity (Pedhazer, 1982). Pairwise plots of the independent variables against $N$ (Xpref $Y$ ) and against one another revealed numerous clear violations of that assumption. We therefore turned to Spearman rank-order correlations.

Table 3 shows the Spearman intercorrelations between the independent variables and the correlations of the latter with $N($ Xpref $Y)$. Values greater than 0.285 or less than -0.285 are significant at the .02 level (two-tailed $t$ test). Significant values are italicized. In our sample of progressions $M D C D$ (modal differentiation between chords) correlated -1.000 with $C T D$ (common-toneness). We therefore dropped the former as completely redundant, so that it does not appear in Table 3. All independent variables correlate positively with $N(X p r e f Y)$. Melodic motion, schema, and scale step form a highly intercorrelated triad. Root-progression schema correlates with both melodic motion and scale step, simply due to constraints in the selection of our sample of progressions. To avoid skips, all IV-I and VI-I progressions had repeated tones in the soprano. We already have pointed out that $M M, O V M$, and $S S$ should intercorrelate in our sample of progressions. In this triad, only SSD and OVMD fail to correlate significantly.

Table 3 shows that the single variable that correlates best with $N(X$ pref $Y)$ is $S C H D$, as Table 1 implies. The Spearman $\varrho$ is 0.619 . Would some additive combination of variables do better? Unfortunately, heteroscedasticity prevented use of standard multiple correlation methods to address this question, even with ranked versions of the difference variables in Table 3. Many plots of the ranks of difference variables against one another also were heteroscedastic. We therefore adopted a novel approach. For preliminary guidance only, we first carried out a multiple regression of the rank of $N(X p r e f Y)$ on the ranks of the independent variables. We made no attempt to interpret the calculated $t$ values for the regression coefficients or the calculated $r$-square. We simply took the coefficients as tentative weights for defining a new variable which we designate $P 8$. To calculate $P 8$, we multiplied the ranks of the eight independent variables by the weights and added the results for each pair of progressions. The values of $P 8$ for the 66 pairs of progressions were then ranked. The Spearman $\varrho$ for $N(X p r e f Y)$ against $P 8$ was 0.766 . Attempts to adjust the tentative weights produced even worse results, suggesting that the weights from multiple regression were as good as anything we could find. 
TABLE 3

Spearman Correlations for Harmonic Variables and Preference Judgments

\begin{tabular}{lrrrrrrrr}
\hline & CTD & SPOSD & BPOSD & OVMD & MMD & SCHD & SSD & CDSD \\
\hline SPOSD & 0.085 & & & & & & & \\
BPOSD & 0.125 & 0.429 & & & & & & \\
OVMD & -0.028 & -0.087 & 0.627 & & & & & \\
MMD & 0.177 & 0.399 & 0.203 & 0.292 & & & & \\
SCHD & 0.551 & -0.011 & 0.067 & 0.192 & 0.776 & & & \\
SSD & -0.097 & -0.278 & 0.134 & 0.194 & 0.489 & 0.658 & & \\
CDSD & -0.191 & -0.367 & -0.812 & -0.615 & -0.311 & -0.270 & -0.064 & \\
N(XprefY) & 0.263 & -0.031 & 0.014 & 0.235 & 0.585 & 0.619 & 0.421 & 0.010
\end{tabular}

NOTE. Italicized values significant at the 0.02 level.

The correlation between $P 8$ and $N(X p r e f Y)$ shows that the variables other than root-progression schema play some relatively minor role in determining judgments of harmonic closure. According to Table 3, however, $S C H D$ correlates highly with $S S D$ and $M M D$ as well as with other variables. This raises the question of which variables (SCHD included) contributing to $P 8$ are really important to the correlation with $N(X p r e f Y)$. To approach this problem, we undertook two kinds of analyses. First, we examined the effects of eliminating single variables or subsets of variables from the eight shown in Table 3. Second, we studied the consequences of adding one variable at a time to SCHD.

We followed our previous procedure of using multiple correlation on rank-ordered variables merely to obtain weights. For each subset of independent variables chosen for examination, we generated a new variable by applying the weights to the appropriate ranks, adding the results, and ranking the final outcomes. The new rank-order variable then was correlated with $N$ (XprefY) by a Spearman $\varrho$.

Table 4 summarizes the results of studying numerous subsets of independent variables. It shows weights for variables retained as predictors of $N(X p r e f Y)$. In each row, the sum of the absolute values of the weights has been normalized to 100 . As was stated previously, a combination of all eight independent variables gave a Spearman $\varrho$ of 0.766 against $N(X$ pref $Y$ ). This result appears in row (a) of Table 4. We then dropped individual independent variables. Leaving out SPOSD alone raised the correlation very slightly to 0.768 (row (b)). Omitting CDSD from the eight predictors produced the largest single decline in $\varrho$. Row (c) shows the outcome. We therefore retained CDSD for the ensuing analyses involving elimination of variables. Despite the fact that $S C H D$ had the highest correlation with $N(X p r e f Y)$, dropping it at this point had no effect (row (d)). 
TABLE 4

Best Predictor Combinations

\begin{tabular}{|c|c|c|c|c|c|c|c|c|c|}
\hline & \multicolumn{8}{|c|}{ Weights for Variables Retained } & \multirow[b]{2}{*}{$\varrho$} \\
\hline & CTD & SPOSD & BPOSD & OVMD & $M M D$ & SCHD & $S S D$ & CDSD & \\
\hline (a) & .3 & 5.2 & -16.2 & 6.9 & 12.7 & 22.3 & -9.6 & 26.8 & 0.766 \\
\hline (b) & 1.9 & & -13.9 & 12.0 & 9.3 & 25.5 & -6.9 & 30.5 & 0.768 \\
\hline (c) & -0.8 & 17.1 & 4.3 & 6.0 & 40.1 & 26.5 & -5.1 & & 0.668 \\
\hline (d) & 14.0 & 6.6 & -16.3 & 7.0 & 26.1 & & 1.9 & 28.0 & 0.765 \\
\hline (e) & 13.0 & 8.4 & -17.6 & 5.7 & 27.6 & & & 27.6 & 0.770 \\
\hline (f) & 15.8 & & -13.7 & 12.9 & 25.7 & & & 31.9 & 0.759 \\
\hline (g) & 16.5 & & & 20.4 & 34.6 & & & 28.4 & 0.740 \\
\hline (h) & & & & 21.3 & 47.8 & & & 30.9 & 0.677 \\
\hline (i) & & & & & & 100.0 & & & 0.619 \\
\hline (j) & & & & 16.4 & & 83.6 & & & 0.632 \\
\hline (k) & & & & 23.8 & & 45.6 & & 30.6 & 0.741 \\
\hline (l) & & -11.4 & & 23.2 & & 34.6 & & 30.7 & 0.750 \\
\hline (m) & -2.0 & 19.6 & -12.8 & & 36.5 & 20.9 & -8.1 & & 0.630 \\
\hline (n) & 9.5 & 22.6 & -15.3 & 0.7 & 51.8 & & & & 0.658 \\
\hline (o) & & & & 20.0 & 14.8 & 32.0 & -5.7 & 27.4 & 0.746 \\
\hline (p) & & & & 21.4 & 17.5 & 31.2 & & 29.9 & 0.741 \\
\hline
\end{tabular}

We then omitted all possible pairs, triplets, and quadruplets of variables, except for $C D S D$. Dropping $S C H D$ and $S S D$ left six variables, CTD, $S P O S D, B P O S D, O V M D, M M D$, and CDSD that gave the highest correlation that we ever found with $N(X p r e f Y)$. Row (e) of Table 4 shows that this correlation was 0.770 . Since $S C H D$ is not among the six remaining predictors, it cannot be necessary for explaining our findings. Dropping SPOSD along with SCHD and SSD (row (f)) lowered the correlation to 0.759 ; thereafter, additional elimination of $B P O S D$ lowered $\mathrm{Q}$ to 0.740 (row (g)). This left four variables, CTD, OVMD, MMD, and $C D S D$, as good predictors of closural judgments. The difference between 0.740 and the maximum obtained correlation of 0.770 is negligible. Eliminating CTD, however, then reduced $\varrho$ to 0.677 (row (h)) from 0.740 . Keeping CTD and dropping any one of the other variables resulted in an even lower correlation. We conclude that CTD is needed along with OVMD, $M M D$, and CDSD in order to give a good account of our results.

The single variable that correlated best with $N(X p r e f Y)$ was $S C H D$. Row (i) of Table 4 merely repeats the fact that $\varrho$ was 0.619 . We then studied the effects of adding single variables, pairs, and triplets of variables to $S C H D$. Adding OVMD as a predictor gave the largest increment in the correlation but raised it trivially to 0.632 (row (j)). Adding CDSD to $S C H D$ and OVMD produced a correlation of 0.741 (row (k)). Row (l) shows that adding SPOSD to those three variables gave a $\varrho$ of 0.750 , just marginally better than that obtained with $C T D, O V M D, M M D$, and 
$C D S D$ as predictors. Although $C D S D$ has no significant correlation by itself with $N(X p r e f Y)$, it always proved a useful predictor in conjunction with other variables.

This analysis indicates that at least three harmonic variables must be invoked to explain our findings. Furthermore, three variables give quite a good account of the preference judgments, as long as one of the three is root-progression schema. We tried other triplets of predictors that did not contain SCHD. The best result was that already shown in row (h) of Table 4.

We found numerous combinations of four, five, six, and seven independent variables that gave correlations with $N$ (Xpref $Y$ ) above 0.740 . If four or more predictors are permitted, many alternative explanations of our results are viable at this stage. Table 4 shows, however, that OVMD and $C D S D$ appear to be necessary but not sufficient to give an acceptable account, whether three or four predictors are used. Dropping these two variables and using CTD, SPOSD, BPOSD, MMD, SCHD, and $S S D$ as predictors gave the results in row $(\mathrm{m})$ of Table 4, where the Spearman $\varrho$ is 0.630 . This observation confirms the apparent importance of outervoice motion and contextual dissonance. Notice also that CDSD always receives a relatively high weighting whenever it appears as a predictor.

Outer-voice motion $(O V M)$ is correlated with melodic motion $(M M)$, which contributes to the former. This fact suggested dropping outer-voice motion in favor of a new variable, interval in the bass. Recall that the latter also contributes to outer-voice motion after a tritone maximum is allowed. We tried two versions of interval in the bass, one with and one without the tritone restriction. Neither gave any advantage over OVM. We also tried omitting the tritone restriction from OVM; again, no advantage ensued.

We have distinguished variables that reflect stylistic schematic structures from those that do not. Completely omitting the former three variables ( $S C H D, S S D$, and CDSD) gave a correlation of only 0.658 (row (n) of Table 4). At least one or more of these three variables is needed for an adequate explanation of our findings. In contrast, eliminating the conventional factors of common-toneness (CTD), soprano position (SPOSD), and bass position (BPOSD) does trivial damage to an explanation of our results, as row (o) shows. Subsequently dropping $S S D$ (row (p)) makes virtually no further difference.

It is worth noting that the pattern of weights in Table 4 changes as different combinations of predictors are used. Whenever MMD, SCHD, or $C D S D$ act as predictors in combination with other variables, their weights tend to be relatively high and stable. In contrast, the weights achieved by each of the other five variables change considerably in combinations with other predictors. These weights may even go negative, 
particularly in the cases of $B P O S D$ and $S S D$. The contrast suggests that $M M D, S C H D$, and $C D S D$ are reliable in their effects.

In summary, the best account of our results requires six harmonic variables (row (e) of Table 4), which do not include harmonic schema. An explanation that is only marginally worse, however, requires just three harmonic variables (row ( $k$ ) of Table 4). Two of them, harmonic schema and contextual dissonance, are grounded in style. This explanation based on OVMD, SCHD, and CDSD has the appeal of parsimony. No other triplet of predictors does as well as this one. Nonetheless, only CDSD proved necessary for an adequate explanation of our results. Dropping $C D S D$ clearly lowered the correlation of $N(X p r e f Y)$ with a weighted combination of the remaining seven variables. Although SCHD correlated the best of all individual variables with $N(X p r e f Y)$, eliminating it from the original battery of eight had no effect on the prediction of $N(X p r e f Y)$. Furthermore, a good prediction of our findings flowed from using just four variables, not including $S C H D$.

Various alternative accounts of our results are possible, once four or more harmonic factors are permitted. Even here some restrictions can be stated. The factors need not include $S C H D$. Eliminating all stylistic variables, however, leaves five predictors that give a somewhat poorer account of the preference judgments than does the most effective triplet of OVMD, $S C H D$, and CDSD. The best predictions using three or more independent variables require invoking $C D S D$ and may require invoking $S C H D$ as well. Style structures always seem to make an important contribution to the perception of harmonic closure. Our evidence also indicates that outervoice motion, along with contextual dissonance, plays a role in determining the perceived strength of harmonic closure. Finally, conventional harmonic variables of soprano position, bass position, common-toneness, and scale step are not necessary to explain our findings. Row (p) of Table 4 shows that eliminating them entirely still allows a good prediction of the results.

In Table 4, all weights but one for BPOS are negative. The single positive weight is the smallest in absolute value of all. The negative weights imply that listeners actually perceived five-three to five-three progressions as more strongly closed than those that involved inversions. We had originally assigned root-position-to-root-position cadences the lowest closural value in order to parallel our analysis of soprano position, because we treated SPOS and BPOS in terms of a common concept of stability. The results in Table 4 speak against this approach. Listeners do not deal with soprano position and bass position in parallel ways. The quantification of $B P O S$ could easily be changed to give six-four to five-three progressions a value of 1 , six-three to five-three progressions a value of 2 , and five-three to five-three progressions a value of 3 . This would invert the signs of all 
correlations in Table 3 that involve BPOS and of all weights in Table 4 for $B P O S$. This change would correspond with beliefs in music theory that root-position-to-root-position chords constitute the most powerful progressions.

The conclusions that we have drawn about the predictive power of different harmonic variables must be qualified. The sample of progressions used here was necessarily limited in scope. We therefore cannot generalize beyond it about the material variables that determine the perception of harmonic closure. Clearly, samples constructed in different ways might produce somewhat different findings. For example, our sample deliberately contained a variety of root-progression structures. Even at that, other progressions exist that are germane to empirical studies about the perception of harmonic closure, e.g., $\mathrm{V}^{6}-\mathrm{I}$ with scale steps $\hat{2}-\hat{1}$ in the soprano, IV-I with $\hat{4}-\hat{3}$ in the soprano, IV-I with $\hat{6}-\hat{5}$, and V-I with $\hat{5}-\hat{3}$. Moreover, one should compare modal-chord configurations of major/minor and minor/minor. One would also want to test progressions from the minor mode. Finally, samples constructed from single root progressions such as V-I would allow more sensitive testing of variables such as soprano position and bass position. Only musically trained listeners might prove responsive to the effects of such factors.

\section{MODELS OF HARMONIC CLOSURE}

Conventional harmonic variables such as soprano position, inversion, or common-toneness are of relatively little general importance for the perception of harmonic closure within our sample of progressions. Stylestructural variables appear more important. If only three variables are allowed, root-progression schema must be among them. Another stylestructural factor, contextual dissonance, seems necessary to explain our results, whether three or more variables are chosen. The features of style structural schemata seem to be stored hierarchically in long-term memory, and their utility may be sensitive to primacy, frequency, and recency of occurrence of the stimuli that fit and maintain them. There is a large and growing literature on the general importance of schemata to cognition and perception and on their specific stylistic relevance to the theory, analysis, and history of music. Although we cannot review the concept here, suffice it to say that the use of schemata in evaluating degrees of harmonic closure seems essential and natural.

The correlational model that we have used has a strong implication. Effective harmonic variables combine additively to determine judgments of closure. We conducted numerous tests of models that included nonadditive interactions. The results were no better than those generated by a purely additive model. Future experiments must be designed, however, 
to explicitly test interactions between harmonic variables. Such interactions may ultimately prove to be important determinants of the perception of harmonic closure.

\section{CONCLUSIONS}

Some psychological solutions to the music-theoretic issues discussed at the beginning of this article now seem possible. Our experiment makes it clear that listeners never equated the harmonic closure of perfect authentic cadences with that of plagal ones. In comparing isolated chord pairs, subjects always judged V-I (in whatever guise) significantly more closed than IV-I. In terms of closure, listeners even preferred III-I progressions with scale steps $\hat{7}-\hat{8}$ in the soprano over IV-I (albeit weakly).

Therefore, the judgment of diverse theorists like Riemann, Schenker, Schoenberg, and Chailley appears to have been right. Moreover, in formulating a generative structural constant like the Ursatz, Schenker appears to have been quite correct to isolate the V-I progression over all others. However, with reference to melody and the Ursatz, listeners showed no closural preference for V-I progressions with scale steps $\hat{2}-\hat{1}$ over those with $\hat{7}-\hat{8}$ or even over those with $\hat{2}-\hat{3}$. In terms of theoretical revision and experimental evidence, the message for Schenkerian theory seems to be that V-I as a generative harmonic constant is empirically tenable, whereas $\hat{2}-\hat{1}$ as a melodic one (in the various Urlinie forms, on whatever level) is not.

Can psychological data help us to specify the intrinsic closural properties of the perfect authentic cadence, as we suggested at the beginning of this article? The variables tested in this experiment provide an answer. Perfect authentic cadences in a major key display the following theoretical properties:

- OVM: a span of a tritone or a perfect fifth;

- CT: only one out of three tones common $(1 / 3)$;

- MM: a melodic span of a major or minor second;

- SPOS: a soprano position of either 5-8 or 3-8;

- BPOS: both chords in root position in the bass;

- MDC: a series of two major chords;

- SS: scale steps of $\hat{2}-\hat{1}$ or $\hat{7}-\hat{8}$;

- CDS: no contextual dissonance.

However, since many other fifth progressions display the same properties except for scale step (SS), we must add the theoretical observation that the dominant never substitutes for any other chord. For it is not the tonic but rather the dominant that is sui generis in diatonic progressions. In functional harmonic theory, where chords built on scale-step roots are reduced to either tonic, subdominant, or dominant function, II can sub- 
stitute for IV (subdominant, up a third from II). And III can substitute for $\mathrm{V}$ (dominant, up a third from III), as our experimental results with III-I showed. Likewise, VI can represent I (tonic, up a third from VI), as in traditional deceptive cadences. But, given a recognized key, the ascending continuation of thirds stops on $\mathrm{V}$, since $\mathrm{V}$ never functionally substitutes for VII. Indeed, just the opposite is the case: VII very frequently functions as dominant (leading-tone chord, down a third from V). In diatonic progressions the dominant and thus the perfect authentic cadence following from it (V-I) are theoretically unique.

The seven variables listed above, however, are not at all closurally equal in weight. Specifically (and surprisingly), Table 4 shows that the conventional theoretical values attached to soprano position, bass position, scale step, and perhaps common-toneness seemed to have counted for very little in the comparisons. To take a few cases in Table 1, had SPOS been perceptually important, listeners would not have equated all three V-I cadences with the root in the bass (preference probabilities of $0.50,0.50$, and 0.60$)$ since each progression displays a different soprano position $(5-8$, 3-8, 5-3). Further, had BPOS been important, subjects would not have evaluated IV-I as more or less equivalent to both IV 6 -I (preference probability of 0.58 ) and to $\mathrm{IV}_{4}^{6}$-I (preference probability of 0.50 ). Moreover, had they attended to $C T$ more carefully, they would not have likened III-I with its common-toneness of $2 / 3$ to IV-I with its common-toneness of $1 / 3$ (preference probability of 0.60 ). Finally, had they truly accorded SS its traditional effect, they would not have perceived V-I with $\hat{2}-\hat{3}$ as more or less the same as V-I with $\hat{2}-\hat{1}$ (preference probability of 0.60 ) or $\hat{7}-\hat{8}$ (preference probability of 0.50 ).

Before concluding that four of the most venerated harmonic variables in music theory-SPOS, BPOS, SS, and possibly $C T$-seem to mean very little in the perception of harmonic closure, we must exercise caution. As already emphasized, our experiment on the closural materials of harmony only tested a relatively small number of progressions in a purposely impoverished environment. Different results might be obtained by preceding the cadences with musically rich contexts. However, harmonic theory has traditionally had strong connections to compositional method and acoustics (rather than to experimental psychology). One should therefore not be surprised that certain conventional tenets of music theory may lack psychological validity for ordinary music listeners. After all, even talented beginning music students have considerable trouble recognizing soprano position and inversion in isolated progressions, as all teachers of aural dictation would readily attest.

The weakness of SPOS, BPOS, CT, and $S S$ as predictor variables in the perception of harmonic closure, however, does not compel the abandonment of these conventional features as analytical devices. The practice of 
style analysis and analytical criticism, where minute compositional (as opposed to psychological or perceptual) differences are of great concern, probably justifies their continuing use in music theory. Perhaps the perception of music by professional musicians may add further justification. In short, as one of us (Rosner, 1988) has pointed out, the goals of music theory need not dovetail everywhere with the interests and claims of the psychology of music. Yet music theory in its analytic explanation of composition should never ignore empirical perceptual data, since ordinary listeners are indispensable to the art of music and thus must ultimately figure in the theoretical disciplines of both style analysis and analytical criticism. Nor should harmonic music theory ignore the positing of new analytical variables like $O V M$ and $M M$, which seemed valuable here in explaining harmonic closure.

In our quest to specify the closural properties of the perfect authentic cadence, what may then be said about the importance of style? Nothing very definitive, it seems. Contextual dissonance $(C D S)$ is certainly necessary to explain the results of the experiment (recall row (c) of Table 4), but the notion of schema $(\mathrm{SCH})$ is not (recall row (d)), unless one opts for strict parsimony. Furthermore, of the stylistic variables, scale step (SS) plays almost no role at all in predicting the results. This is clear in rows (e), (f), and (g) of Table 4, where the absence of SSD produces trivial changes in the correlations.

The perfect authentic cadence (V-I) is a strong progression partly because its intrinsic parametric properties make it perceptually unique: given an established tonality, it never substitutes for any other progression. Moreover, the schematic uniqueness of V-I lends itself perfectly to the recurrent syntactic needs of tonality by enabling listeners to parse and to store diverse chunks of tonal musical forms. ${ }^{1}$

\section{References}

Apel, W. (Ed.) Harvard dictionary of music. London: W. \& G. Foyle Limited, 1944.

Arnold, D. (Ed.) The new Oxford companion to music. Oxford: Oxford University Press, 1983.

Balzano, G. J., \& Liesch, B. W. The role of chroma and scalestep in the recognition of musical intervals in and out of context. Psychomusicology, 1982, 2, 3-31.

Bernstein, M. An introduction to music. London: Pitman and Sons, 1937.

Bregman, A.S., \& Campbell, J. Primary auditory stream segregation and perception of order in rapid sequence of tones. Journal of Experimental Psychology, 1971, 89, 244249.

1. This work was largely carried out while Eugene Narmour was a Visiting Fellow at Wolfson College, Oxford. We thank the IBM UK Science Centre, Winchester, England, for facilities for preparing tapes. We are particularly indebted to J. B. Pickering for his help there. Helena Stoward made many useful suggestions about an earlier version of this paper. 
Bregman, A. S., \& Dannenbring, G. L. The effect of continuity on auditory stream segregation. Perception and Psychophysics, 1973, 13, 308-312.

Butler, D. Describing the perception of tonality in music: A critique of the tonal hierarchy theory and a proposal for a theory of intervallic rivalry. Music Perception, 1989, 6, 219-242.

Butler, D. Response to Carol Krumhansl. Music Perception, 1990, 7, 325-338.

Castellano, M. A., Bharucha, J. J., \& Krumhansl, C. L. Tonal hierarchies in the music of North India. Journal of Experimental Psychology: General, 1984, 113, 394-412.

Chailley, J. Historical treatise of harmonic analysis (S. Kleinman, Trans.). Paris: Alphonse Leduc, 1986. (Original work published 1977.)

Christ, W., \& DeLone, R. Introduction to materials and structure of music. Englewood Cliffs, NJ: Prentice-Hall, 1975.

Cooper, G., \& Meyer, L. B. The rhythmic structure of music. Chicago: University of Chicago Press, 1960.

Cuddy, L. L., \& Badertscher, B. Recovery of the tonal hierarchy: Some comparisons across age and levels of musical experience. Perception and Psychophysics, 1987, 41, 609620.

Harder, P. O. Harmonic materials in tonal music. Part 1. Boston: Allyn \& Bacon, 1977.

Krumhansl, C. L. The psychological representation of musical pitch in a tonal context. Cognitive Psychology, 1979, 11, 346-374.

Krumhansl, C. L. Perceptual structures for tonal music. Music Perception, 1983, 1, 28-62.

Krumhansl, C. L. Tonal hierarchies and rare intervals in music cognition. Music Perception, 1990, 7, 309-324.

Krumhansl, C. L., \& Shepard, R. N. Quantification of the hierarchy of tonal functions within a diatonic context. Journal of Experimental Psychology: Human Perception and Performance, 1979, 5, 579-594.

McAdams, S., \& Bregman, A. S. Hearing musical streams. Computer Music Journal, 1979, 3, 26-43.

McHose, A. I. Basic principles of the techniques of 18th and 19th century composition. New York: Appleton-Century-Crofts, 1951.

Meyer, L. B. Emotion and meaning in music. Chicago: University of Chicago Press, 1956. Meyer, L. B. Explaining music. Berkeley: University of California Press, 1973.

Meyer, L. B. Style and music. Philadelphia: University of Pennsylvania Press, 1989.

Morris, R. O. The Oxford harmony, Vol. 1. London: Oxford University Press, 1946.

Narmour, E. Beyond Schenkerism. Chicago: University of Chicago Press, 1977.

Narmour, E. The analysis and cognition of basic melodic structures. Chicago: University of Chicago Press, 1990.

Pedhazer, E. Multiple regression in behavioral research (2nd ed.). New York: Holt, Rinehart, \& Winston, 1982.

Piston, W. Harmony. New York: Norton, 1978.

Rameau, J.-P. Nouveau système de musique theoretique. Paris: J. B. C. Ballard, 1726.

Randel, D. (Ed.) The new Harvard dictionary of music. Cambridge, MA: Belknap, 1986.

Reynolds, W. H., with Warfield, G. Common-practice harmony. New York: Longman, 1985.

Riemann, H. Theory of harmony (Book III of History of music theory [W. C. Mickelsen, Trans.]). Lincoln, NE: University of Nebraska Press, 1977. (Original work published 1898)

Rosner, B. S. Music perception, music theory, and psychology. In E. Narmour \& R. Solie (Eds.), Explorations in music, the arts, and ideas. Stuyvesant, NY: Pendragon, 1988.

Sadai, Y. Harmony in its systemic and phenomenological aspects. Jerusalem: Yanetz, 1980.

Sadie, S. (Ed.) The new Grove dictionary of music and musicians. London: Macmillan, 1980.

Schenker, H. Harmony (E. M. Borgese, Trans.). Chicago: University of Chicago Press, 1954. (Original work published 1906) 
Schenker, H. Free composition (E. Oster, Trans.). New York: Longman, 1979. (Original work published 1935, 1956)

Schoenberg, A. Structural functions of harmony. New York: Norton, 1954.

Tchaikovsky, P. I. Guide to the practical study of harmony (E. Krall \& J. Leibling, Trans.). Canoga Park, CA: Summit, 1970. (Original work published 1871)

Toutant, W. Functional harmony. Belmont, CA: Wadsworth, 1985.

White, J. D. The analysis of music. Metuchen, NJ: Scarecrow, 1984. 